\title{
Limited onboard amenities
}

Published at www.cmaj.ca on May 27, when Expedition 20/21 launched.

$\mathrm{S}$ ince the dawn of human space flight nearly a half-century ago, about 500 people have lived and worked in space.

Each of us have had the opportunity to fly in space at least once. We can attest that space flight is a challenging, exhilarating and fulfilling human endeavour.

But adaptation to space flight is not easy. Since we are earthlings, it takes time for our bodies to adapt to this new environment. Some physiologic systems, such as the cardiovascular and neurovestibular systems, adapt quickly. For instance, we experience a headward shift of blood and extravascular fluid soon after arriving in orbit, which results in "puffy faces," distended jugular veins, headaches and persistent nasal congestion.

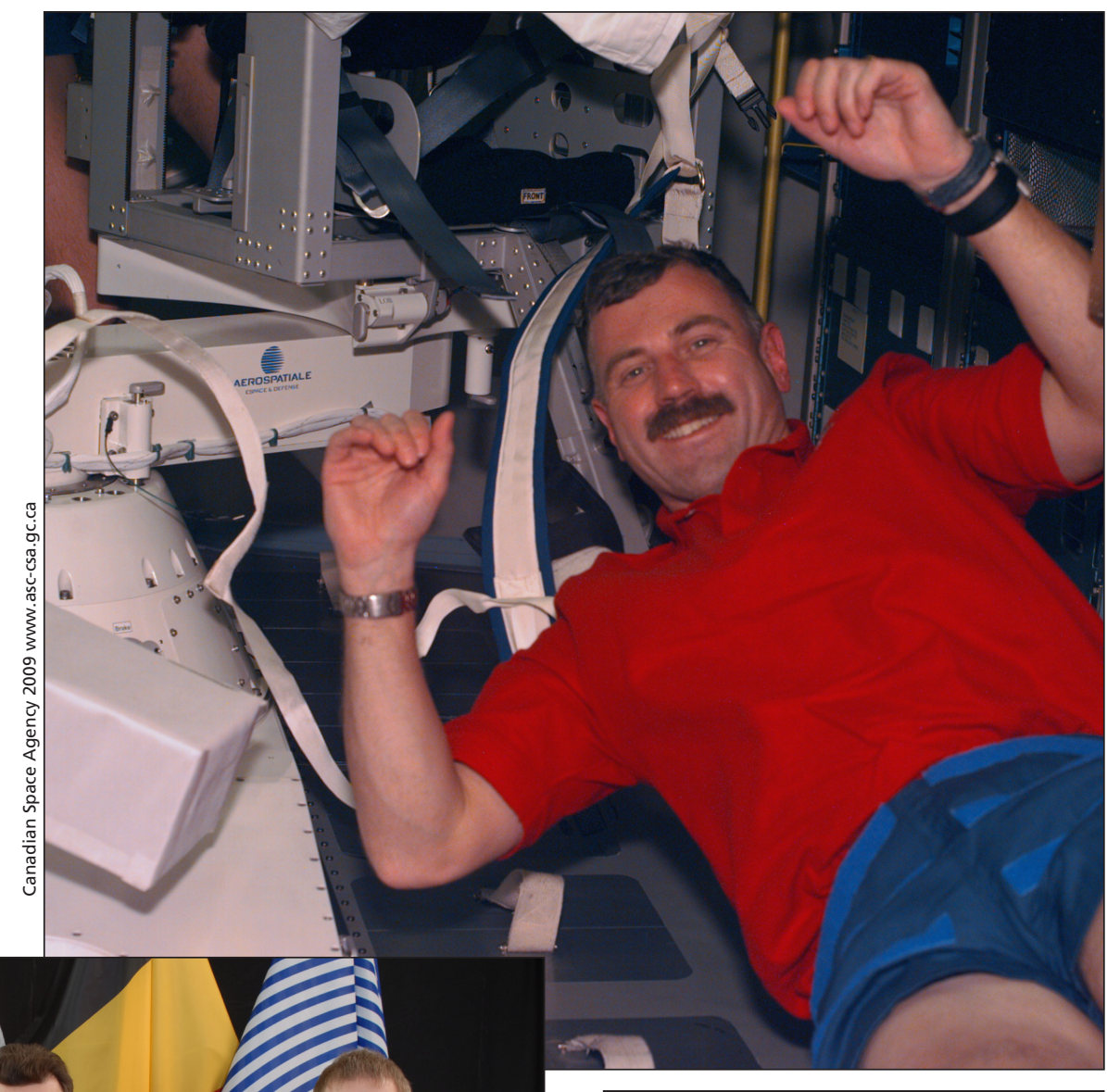

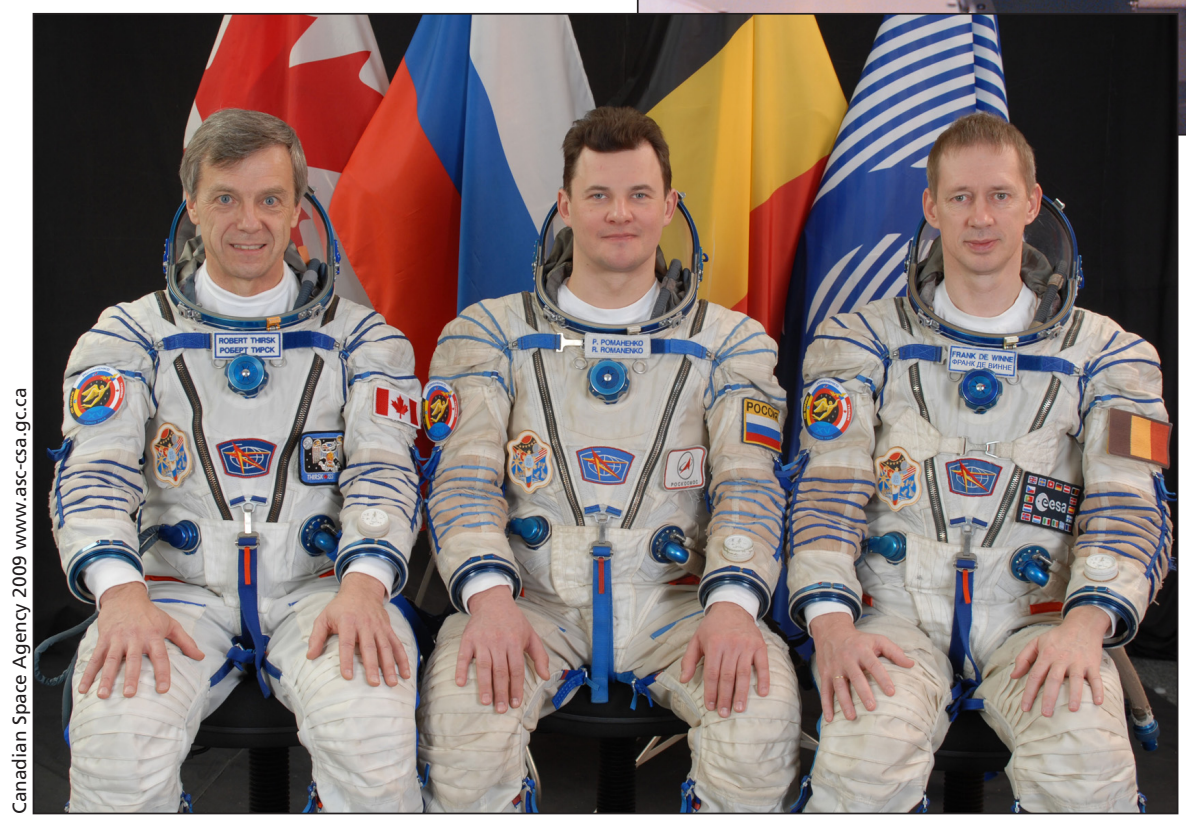

Canadian astronaut Dr. Robert Thirsk (left), along with Roman Romanenko and Frank De Winne, are crew members for Expedition 20/21. With a 6-month schedule, the expedition will mark the longest period that a Canadian astronaut has been in space, and the first time that the Canadian space program has had a participant in a longduration mission. Thirsk and his colleagues are scheduled to be launched aboard a Soyuz rocket from Baïkonur, Kazakhstan.
Canadian astronaut Dr. Dave Williams takes a break between Neurolab experiments on mission STS-90.

For some of us, the first few days in space can be uncomfortable as we struggle with motion sickness. Once this is resolved, the notions of $u p$ and down become irrelevant. We are just as comfortable with our feet on the ceiling as on the floor, or even floating in the middle of the cabin.

In a weightless environment, our intervertebral discs expand and our spinal curvatures are reduced, resulting in a height increase of 4-8 centimetres. That's pretty cool for vertically challenged astronauts! The downside is that many astronauts are bothered by low back pain throughout flight, presumably due to stretching of vertebral ligaments or spinal nerve roots. 
Other adaptive processes take longer. Several weeks in space must pass before we become adept in our movements about the spaceship. Translating from one place to another is as simple as pushing off a wall and flying like superman to our destination. Our leg musculature has little function in this kind of locomotion and, with time in space, will atrophy.

Onboard amenities are limited to those that require little mass, volume, power and consumables. We have no washing machine, no fridge or freezer. We eat processed food instead of fresh fruits and vegetables; we use sleeping bags instead of king-size beds. We endure loud ambient noise from the onboard machinery that must continuously operate to keep our spacecraft operating nominally. The life-support system keeps us alive by providing a pressurized atmosphere, purified air to breathe and humidity control. It even recycles our sweat and urine into potable water.

Daily grooming that we took for granted on Earth now becomes an exercise in choreography and patience. Without an onboard shower or bathtub, we do the best we can with washcloths to wipe away built-up grime. In a

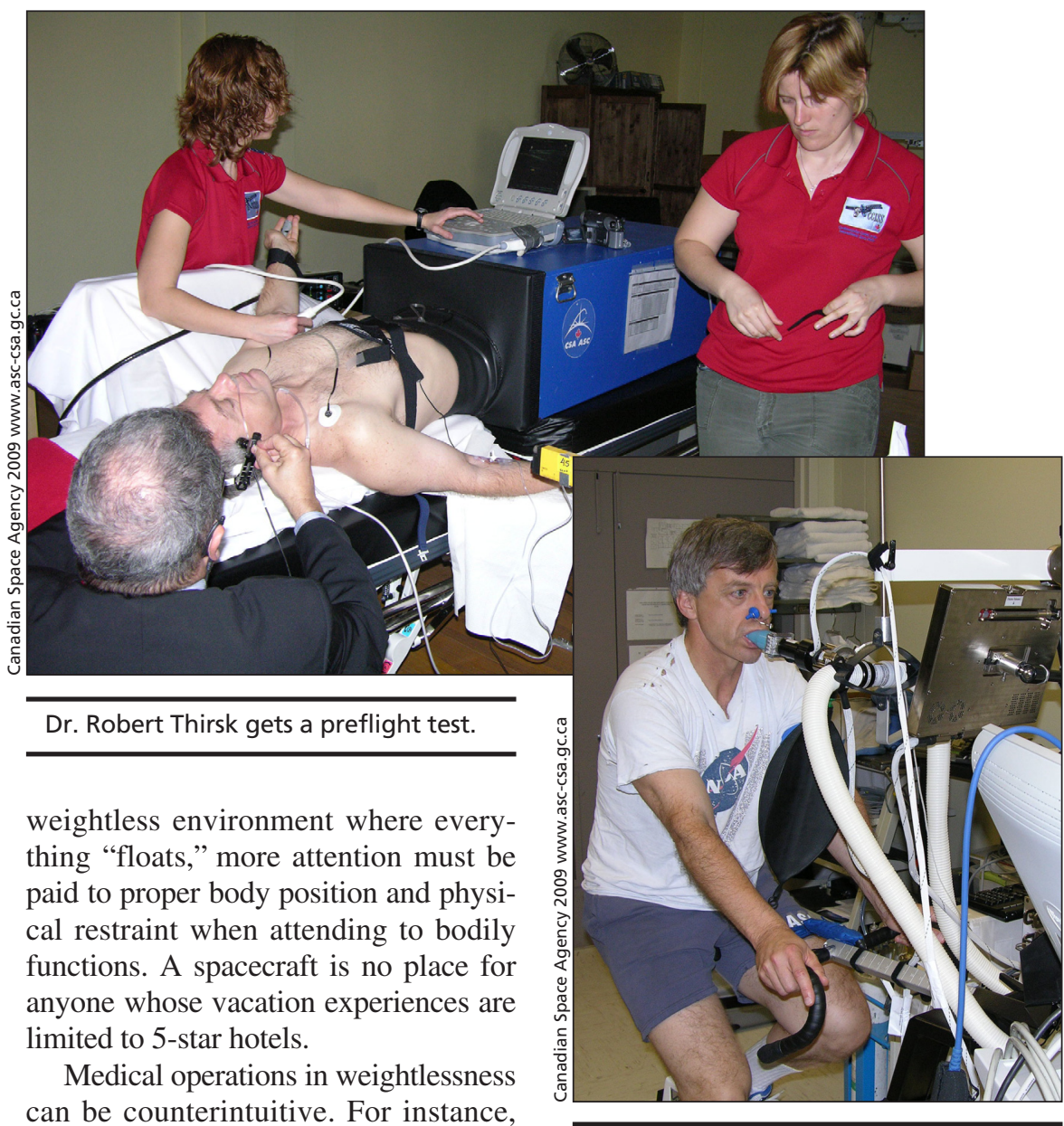
protocols for cardiopulmonary resusci-

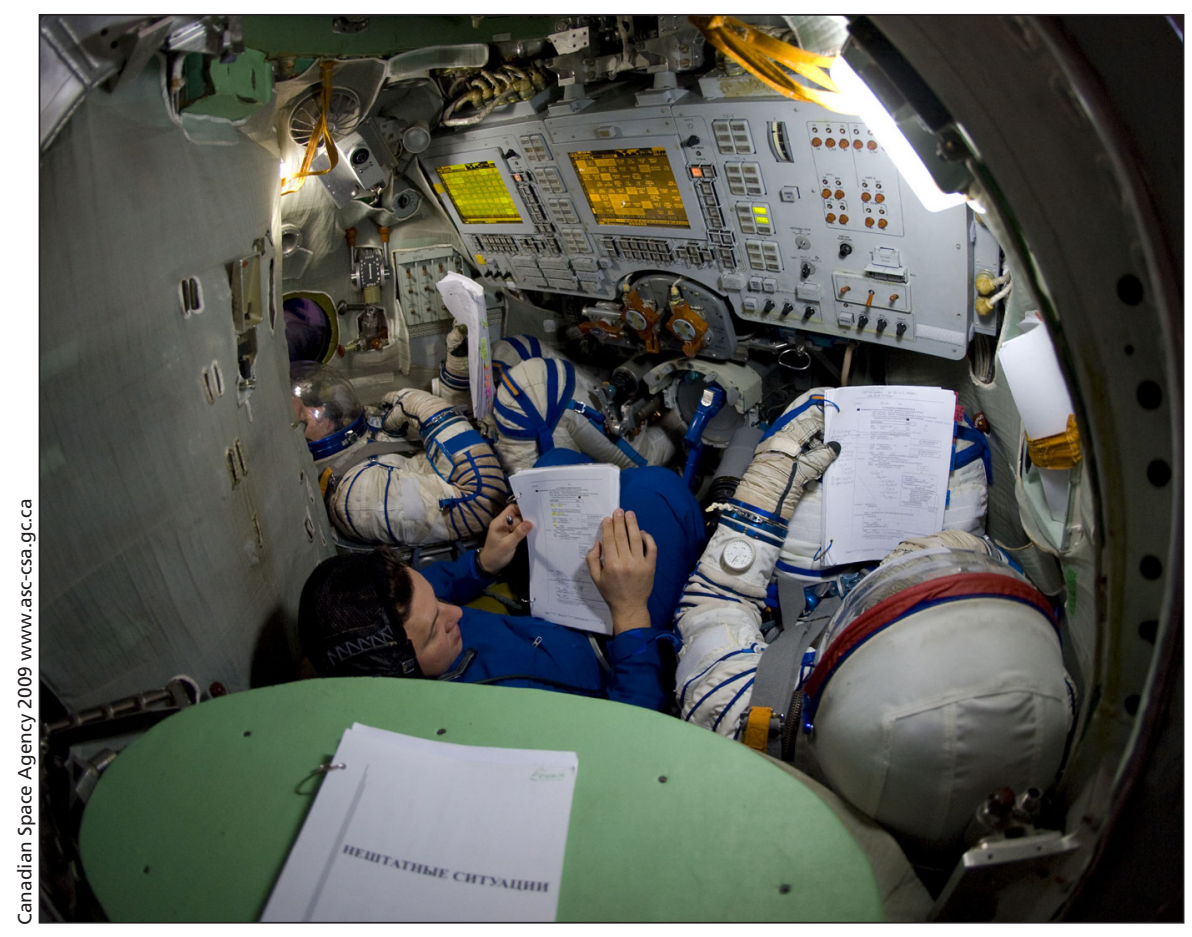

Dr. Robert Thirsk training in a Soyuz Capsule simulator.
Dr. Robert Thirsk training for Expedition 20/21.

tation and advanced life support must be modified to what can practically be accomplished in a weightless environment. We can no longer count on the assistance of gravity to restrain the subject and operator, nor to flow intravenous fluids into the subject's veins. Living in space requires us to think and act in different ways. - Dr. David Williams, Dr. Robert Thirsk, Dr. André Kuipers and Dr. Chiaki Mukai

DOI:10.1503/cmaj.090925

CMAJ invites contributions to "Dispatches from the medical front," in which physicians and other health care providers offer eyewitness glimpses of medical frontiers, whether defined by location or intervention. Submissions, which must run a maximum 700 words, should be forwarded to: wayne.kondro@cmaj.ca. 